\title{
Uma Nova Abordagem para Extração de Parâmetros do Modelo de Seguidores Visuais Baseado em NMPC aplicado ao Husky $\mathrm{UGV}^{\star}$
}

\author{
Iago José P. B. Franco, ${ }^{*}$ Tiago T. Ribeiro, ${ }^{*}$ \\ André Gustavo S. Conceição* \\ * LaR - Laboratório de Robótica \\ Departamento de Engenharia Elétrica e Computação \\ Universidade Federal da Bahia \\ Salvador, Bahia, Brasil \\ E-mails: pattasiago@gmail.com,tiagotr@ufba.br, \\ andre.gustavo@ufba.br
}

\begin{abstract}
Images captured by cameras, in order to perform image-based control, require processing to extract useful information in the presence of noise and obstacles. The problem of visual path following faces several drawbacks, more precisely in detecting the marks representing the route to be followed. Handling faults along the path on non-homogeneous floors and extracting parameters accurately, are some of the challenges encountered. In this paper, an image detection and parameter extraction system is proposed for path following using NMPC em (Nonlinear Model Predictive Control) controller. To circumvent the above obstacles, the visual path is approximated by a quadratic function. The proposed algorithm was embedded in the Husky UGV (Unmanned Ground Vehicle) mobile robot and compared with the original approach. Experimental results with the use of metrics such as IAE and TV demonstrate the superiority of the proposed new algorithm.

Resumo: As imagens captadas por câmeras, com o intuito de realizar controle baseado em imagem, necessitam de processamentos para a extração de informações úteis na presença ruídos e obstáculos. O problema do seguimento de caminhos visuais depara-se com diversas inconveniências, mais precisamente na detecção das marcas que representam a rota a ser seguida. Falhas no caminho em pisos não-homogêneos e extração de parâmetros de controle com elevada precisão, são alguns dos desafios encontrados. Neste artigo, é proposto um sistema de detecção e extração de parâmetros por imagens, para o seguimento de caminhos utilizando controlador NMPC(Nonlinear Model Predictive Control). Para contornar os empecilhos supracitados, faz-se uma aproximação do caminho visual através de uma função quadrática. O algoritmo proposto foi embarcado no robô móvel Husky UGV(Unmanned Ground Vehicle) e comparado com a abordagem original. Resultados experimentais junto a utilização de métricas como IAE e TV demonstram a superioridade do novo algoritmo proposto.
\end{abstract}

Keywords: Computer Vision; Path Following; NMPC; Visual Control.

Palavras-chaves: Visão Computacional; Seguimento de Caminho; NMPC; Controle Visual.

\section{INTRODUÇÃO}

Com o desenvolvimento contínuo de robôs autônomos e um maior poder de decisão baseados em sistemas de visão, que tendem a aumentar a gama de aplicações potenciais, diversos são os estudos voltados à detecção e navegação através de caminhos visuais ( $\mathrm{Lu}$ et al., 2014; Mammeri et al., 2014; Chen et al., 2010).

Atualmente, com o projeto de soluções voltadas à área de visão computacional e o avanço das pesquisas, certos

* This project has received funding from SEPIN/MCTI under the 4 th Coordinated Call BR-EU in CIT and from the European Union's Horizon 2020 research and innovation programme under the Grant Agreement No 777096 . algoritmos eficientes para detecção de características foram validados em diferentes tipos de cenários, tais como industrial (Gorbunov et al., 2018), transportes (Tianqi, 2017) e agricultura (Arakeri et al., 2017). A utilização destes algoritmos em técnicas de seguimento de caminhos visuais permite tornar as soluções menos sensíveis à perturbações variadas, como: imperfeições no ambiente de navegação, robô móvel e do próprio sistema visual. É possível encontrar na literatura métodos de extração de caminhos que utilizam técnicas de detecção por bordas, blobs (Corke, 2011), segmentação por cores (Sun et al., 2006), dentre outros. Os principais usos de tais abordagens estão incluídos nos sistemas: ITS (Intelligent Transportation Systems) 
e ADAS (Advanced Driver Assistance) (Kim, 2008; Kuo et al., 2011).

A detecção de marcas para identificação do caminho por vezes não é uma tarefa tão simples, pois o robô eventualmente encontra alguns problemas, no que diz respeito à luminosidade, falhas no percurso ou pouco contraste das marcas que representam a rota a ser seguida (Mammeri et al., 2014).

Em muitos trabalhos, a estimação de funções para representação do caminho detectado, é ferramenta frequentemente utilizada. Segundo (Lu et al., 2014), os estudos tentam encontrar um equilíbrio entre representar caminhos mais complexos ou representá-los mais precisamente. Por exemplo, para linhas retas é frequente o uso da transformada Hough (Corke, 2011), abordagem mais simplista, Splines (Wang et al., 2004) consegue lidar com caminhos mais complexos, porém exigem mais tempo de processamento. Já, curvas parabólicas (Lu et al., 2014), são consideradas um meio termo entre precisão e complexidade, sendo alvo de investigação no presente trabalho.

O presente trabalho tem como objetivo contribuir com uma proposta de algoritmo para extração e detecção de parâmetros que representam o caminho a ser seguido, aplicando-o no controlador NMPC proposto por (Ribeiro, 2018a), visando incrementar os índices de robustez da técnica sem comprometer em demasia o custo computacional. A estratégia aqui proposta visa representar melhor o caminho e sanar alguns problemas encontrados no algoritmo anterior, como: navegação em pisos de coloração nãohomogênea, falhas no caminho de referência e imprecisão nos cálculos da curvatura visual.

O artigo está organizado da seguinte forma: Na Seção 2 o problema é formalizado. Na Seção 3 encontram-se os principais fundamentos das técnicas utilizadas. Na Seção 4 estão descritos os materiais, métodos, e resultados experimentais e na Seção 5 são fornecidas as principais conclusões.

\section{FORMALIZAÇÃO DO PROBLEMA}

Os elementos necessários à modelagem do problema de seguimento de caminhos visuais, tal como proposto por (Ribeiro, 2018a) são apresentados na Figura 1. Neste modelo, para um perfil de velocidade linear $v$ pré-especificado, os estados (idênticos às saídas no presente caso), são parâmetros extraídos a partir de um algoritmo de visão computacional a cada iteração, e a entrada é o erro entre a velocidade angular do robô e de um veículo virtual posicionado no horizonte visual. Tal modelo é definido da seguinte forma:

$$
\begin{aligned}
& \mathbf{u}_{\mathbf{e}}=\omega-c(s) \frac{(v+\omega Z)}{\cos \theta_{r}} \\
& \mathbf{x}_{e}=\left[\begin{array}{c}
\dot{z} \\
\dot{\theta}_{r}
\end{array}\right]=\left[\begin{array}{c}
\omega H+(\omega H+v) \tan \left(\theta_{r}\right) \\
\mathbf{u}_{e}
\end{array}\right] .
\end{aligned}
$$

Onde $\omega$ é a velocidade angular em $\mathrm{rad} / \mathrm{s}, v$ velocidade linear em $\mathrm{m} / \mathrm{s}$. Os parâmetros extraídos da imagem $H, Z$ em pixel e $\theta_{r}$ em rad. A função $c(s)$ representa o caminho de curvatura e neste caso, deve satisfazer:

$$
\dot{c}=\frac{d c}{d s} \frac{(v+\omega z)}{\cos \theta_{r}}
$$

Observa-se que, para o sucesso das implementações de controladores para este modelo, é necessária a aquisição de medidas precisas de curvaturas diretamente no plano da imagem.

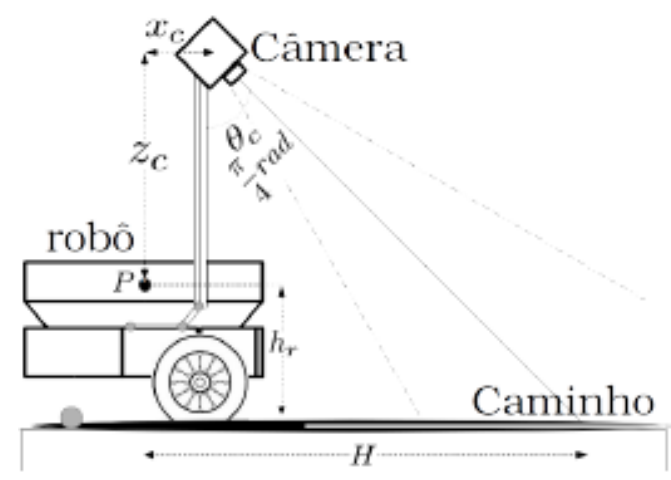

(a) Vista Lateral

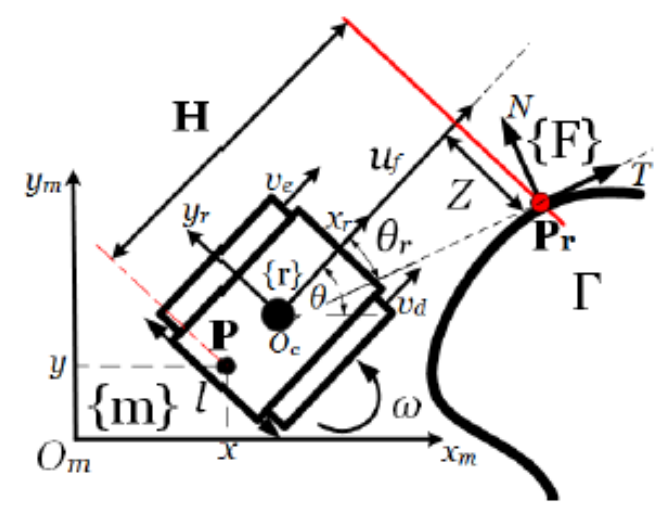

(b) Sistema de Coordenadas.

Figura 1. Seguimento de caminhos visuais (Ribeiro, 2018a).

$\mathrm{Na}$ proposta original, apesar das vantagens obtidas em desempenho e esforço computacional, o algoritmo ainda possui algumas limitações, tal como impossibilidade de tratar descontinuidades no caminho de referência e variabilidade de cadência visual e iluminação.

(Ribeiro, 2018b) resolve parcialmente tais problemas através da inclusão do método de Otsu na abordagem original, tendo como contrapartida a exigência de que o cénario fosse mais controlado, no sentido de haver objetos com cores homogêneas e com diferentes contrastes para que a identificação do caminho pelo algoritmo adaptativo não fosse comprometida. Um exemplo em que tal proposta não se aplica é o próprio cenário visto na Figura 6(a), que possui pisos de cores não homogêneas separados por rejuntes, além de conter o próprio caminho representado pela fita amarela.

Além disso, em nenhuma das abordagens anteriores, existe uma função que estime o caminho a ser percorrido, gerando problemas com a exatidão no cálculo da curvatura. Nestes dois casos, a estimação é feita utilizando as coordenadas de um círculo (ver Figura 2), de modo que, a depender da deformação do caminho visual, serão fornecidos valores 


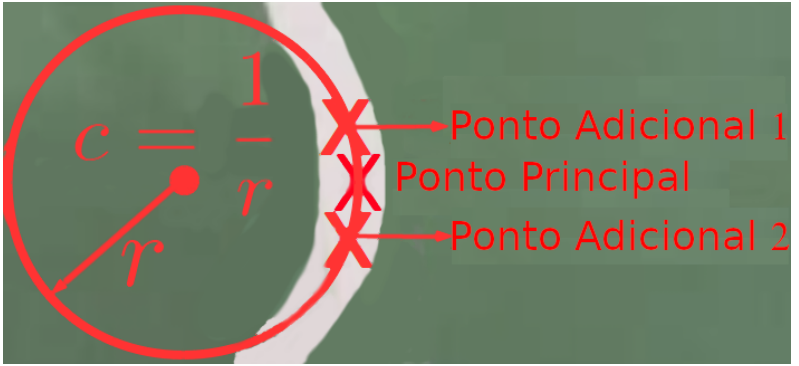

Figura 2. Cálculo da Curvatura na abordagem Original.

errôneos ao controlador, forçando que o sensor de visão seja utilizado muito próximo ao caminho, prevenido que qualquer trepidação no robô não gere valores absurdos de curvatura.

Tais problemas se somam à sensibilidade da calibração dos parâmetros visuais, etapa de maior complexidade quando se trata de plataformas de grande porte como o o robô móvel Husky $U G V^{1}$ utilizado no presente trabalho.

Resumidamente, o presente trabalho visa solucionar os problemas listados a seguir:

- Navegação ao longo de caminhos visuais arbitrários e descontínuos em cenários não-homogêneos.

- Imprecisões nos cálculos da função curvatura visual.

- Aplicação em robôs de grande porte que tendem a reforçar perturbações mecânicas, dificultando a extração de parâmetros visuais.

\section{PROCESSAMENTO DE IMAGEM E ESTIMAÇÃO DA FUNÇÃO CAMINHO}

O algoritmo de visão computacional implementado neste trabalho visa melhorar a representação do caminho com o objetivo de aumentar o desempenho da aplicação, junto ao controlador NMPC.

O algoritmo tem como entrada frames de $640 \times 480$ pixels em espaço de cores RGB(Red Green Blue).

A etapa de classificação e segmentação da linha que representa o caminho na imagem, foram obtidos através da abordagem de segmentação por cores (Sun et al., 2006). A fim de obter uma melhor representatividade das cores na imagem, o espaço de cores dos frames foi convertido de RGB para HSV(Hue Saturation Value).

A Figura 3 mostra a representação gráfica desse espaço de cores (HSV), onde $\mathrm{H}$ corresponde a uma cor que está descrita num valor entre $\left[0^{\circ} 360^{\circ}\right]$, S a pureza da cor variando de [0 1], onde 0 representa o cinza e 1 a cor pura, e V o brilho da cor variando de $\left[\begin{array}{ll}0 & 1\end{array}\right]$ onde 0 corresponde ao preto e 1 a cor com mais brilho. Como a cor do caminho é predominamente amarela, o valor utilizado para a etapa de classificação, varia dentro de uma faixa tanto para $\mathrm{H}$ quanto para $\mathrm{S}$ e $\mathrm{V}$, podendo assim lidar com pequenas diferenças de iluminação e tonalidade.

\begin{tabular}{llcc}
\hline 1 Husky & unmanned & ground & vehicle. \\
https://www.clearpathrobotics.com/husky-unmanned-ground-
\end{tabular}
vehicle-robot, acessado em: 10/04/2019

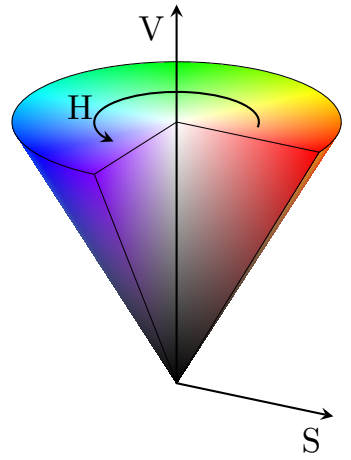

Figura 3. Cone de Cores HSV

Após classificar a imagem, o resultado é uma máscara binária (preto e branco), Figura 4(a), onde os pixels brancos correspondem ao caminho na imagem. Utilizandose desses pontos, uma curva do segundo grau do tipo $x=a y^{2}+b y+c$ é estimada para representar o seguimento. Então, a partir da curva, escolhem-se 8 coordenadas, Figura 4(b), de iguais espaçamentos de acordo com o correspondente da dimensão $\mathrm{H}$ no plano da imagem, e em todos os pontos é calculada uma curvatura a partir da seguinte equação:

$$
\frac{1}{C}=\frac{\left(1+\left(\frac{\mathrm{d} x}{\mathrm{~d} y}\right)^{2}\right)^{\frac{3}{2}}}{\left|\frac{\mathrm{d}^{2} x}{\mathrm{~d} y^{2}}\right|}
$$

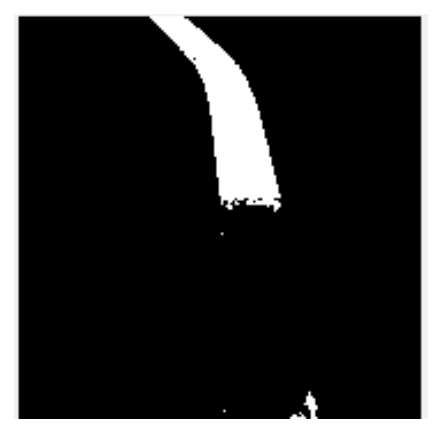

(a) Máscara Binária

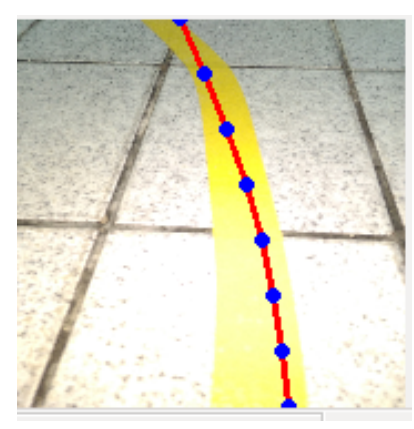

(b) Curva que representa o caminho.
Figura 4. Detecção do Caminho.

Após calcular a curvatura, calcula-se a ângulo entre a $4^{\mathrm{a}}$ coordenada(dentre as 8, de baixo para cima) e o eixo das ordenadas com relação ao centro da camera para obter a orientação de referência, Figura 5. Com tais características extraidas é possível fornecer as entradas adequadas ao preditor do algoritmo de NMPC.

\section{RESULTADOS EXPERIMENTAIS}

Para avaliar o desempenho da nova abordagem, comparouse esta, com a técnica introduzida em (Ribeiro, 2018b) porém modificada, utilizando o espaço de cores HSV como parâmetro para classificar a imagem, e não mais o método de Otsu. Um cenário com um caminho de cor amarela arbitrário, com função curvatura variável e com falhas propositais, ver Figura 6(a), foi montado num local plano, onde partes do trajeto foram removidos para fins 


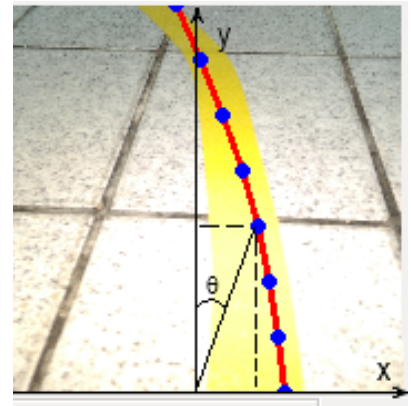

Figura 5. Cálculo do ângulo de orientação $\theta$.

de análise. Os algoritmos desenvolvidos foram embarcados no robô diferencial Husky $U G V$, figura 6(b), junto a uma webcam Microsoft ${ }^{\circledR}$ LifeCam HD-3000 utilizada como sensor de visão com a seguinte pose relativa ao centro de massa do robô: $x=0,25 \mathrm{~m} ; y=0 \mathrm{~m} ; z=0,36 \mathrm{~m}$; $\theta_{c}=\frac{\pi}{4} \mathrm{rad}$.

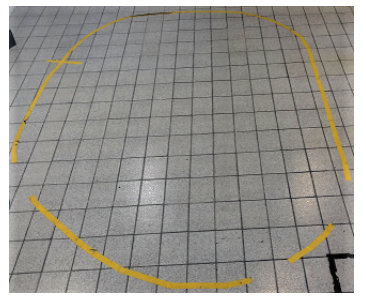

(a) Caminho de referência

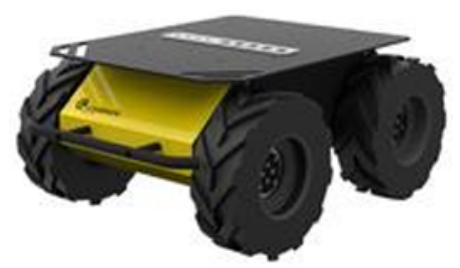

(b) Husky UGV.
Figura 6. Plataforma experimental.

O controlador NMPC, mantendo a nomenclatura apresentada em (Ribeiro, 2016), considera $T_{p}=T_{c}=3 T_{s}$, com $T_{s}=0,2 \mathrm{~s}, v=0,2 \mathrm{~m} / \mathrm{s}$ e os seguintes parâmetros de sintonia: $Q_{i}=\operatorname{diagonal}(0,1 ; 0,1) ; R_{i}=0,01$. As restrições elementares foram definidas como:

$$
\left|u_{1}\right| \leq 0,2 \mathrm{~m} / \mathrm{s} ;|z| \leq 0,20 \mathrm{~m} ;\left|\theta_{e}\right| \leq 0,5 \mathrm{rad} ;
$$

As Figuras 9(a) e 9(b) fornecem os erros de seguimento de caminho visual das abordagens original e nova, respectivamente $^{2}$. Observa-se que com a nova abordagem, a perturbação incluída transversalmente ao caminho foi rejeitada satisfatoriamente e as falhas adicionadas ao caminho foram desperazadas, comprovando a eficácia da interpolação geométrica realizada. Por outro lado, a abordagem original, ainda que regulando a perturbação transversal, não consegue prosseguir quando existem falhas no caminho.

Os resultados coletados a partir da perspectiva dos controladores, podem ser observados nas Figuras 9(c) e 9(d), onde é possivel constatar que com a abordagem original, surgem variações abruptas para a regulação da primeira perturbação, o que tende a desgastar mais facilmente os atuadores.

A evolução temporal das posturas ao longo dos experimentos são fornecidos nas Figuras 9(e) e 9(f), sendo possível confirmar que a abordagem anterior falha por volta de 35 $\mathrm{s}$ enquanto a nova segue completamente o caminho visual especificado, completado em cerca de $60 \mathrm{~s}$.

\footnotetext{
2 Estes resultados podem ser melhor visualizados em vídeo disponível em: https://youtu.be/GbFwCinpTEU
}

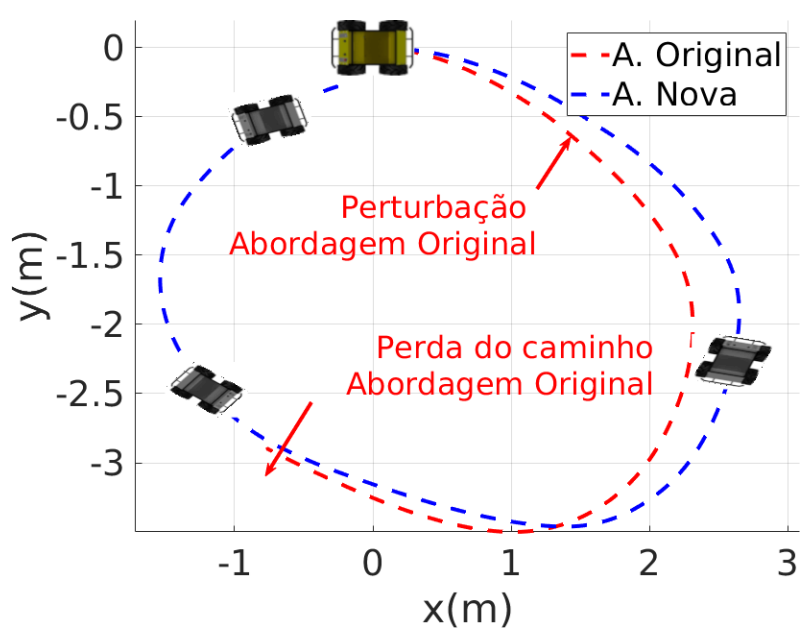

Figura 7. Posições instantâneas

As Figuras 9(g) e 9(h) mostram os valores de curvatura calculados ao longo do tempo. É possivel perceber, destacadamente para o novo algoritmo, a partir do $t=40$, as curvas acentudas que o robô se depara, e o controlador conseguindo agir de maneira satisfatória fazendo o robô manter-se no caminho.

A diferença de performance, se dá pela diferença de abordagem. No algoritmo antigo, foi implementado uma técnica baseada em blobs(regiões conectadas), sem estimar função caminho, onde são extraídas as coordenadas principais que servem de entrada para o controlador, logo quando há um trecho faltante o algoritmo perde as referências do caminho e finaliza o processo parando o que está a fazer. Por outro lado, o novo algoritmo estima uma curva na qual fornece parâmetros mais precisos ao preditor, logo, como é possivel ver na figura 4(a), mesmo com um forte ponto de luz que faz com o que o caminho não seja identificado, apenas um trecho visível permitiu o algoritmo estimar uma função para o caminho. A Figura 8 ilustra alguns cenários encontrados nos dois experimentos que confirmam a presente análise.

Finalmente, compara-se as abordagens através de duas métricas quantitativas, mais especificamente, utiliza-se a integral do erro absoluto $(I A E)$ e a variação de controle total $(T V)$. O índice $I A E$, calculado por $\int_{0}^{T_{E N D}}|e(t)| d t$, é muito utilizado para comparação de desempenho de estratégias distintas em experimentos semelhantes, por outro lado o índice $T V$, calculado por $\sum_{k=0}^{k_{E N D}} \mid u(k)-$ $u(k-1)$, objetiva avaliar o efeito dos rú́dos nos sinais de controle.

Para o problema em questão, os erros $\left(z\right.$ e $\left.\theta_{r}\right)$ e as ações de controle $(v$ e $\omega)$, para o período em que as duas abordagens funcionaram, geram os dados ilustrados na Tabela 2. Todos os índices relacionados diretamente com a implementação do NMPC, ou seja, excetuandose o índice $v_{T V}$ que não é controlado, possui valores inferiores com a nova abordagem, confirmando-se assim a sua superioridade.

\section{CONCLUSÕES}

Este trabalho apresentou uma nova forma de identificar e extrair parâmetros de um caminho a ser seguido, di- 


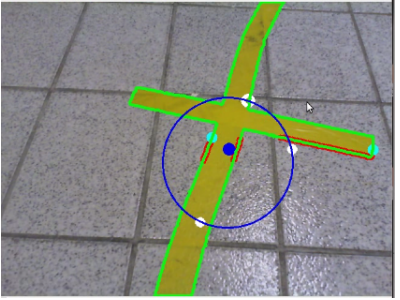

(a) Perturbação - A. Original.

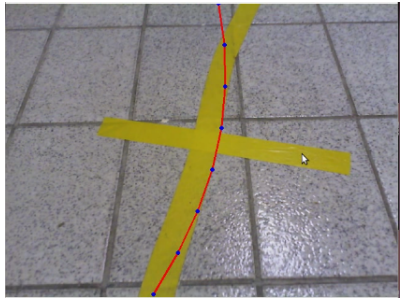

(b) Perturbação - A. Nova.

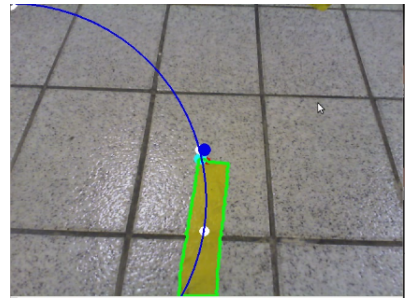

(c) Falhas - A. Original.

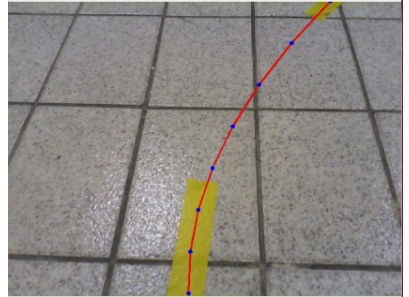

(d) Falhas - A. Nova.

Figura 8. Alguns dos cenários encontrados no plano da imagem para as duas abordagens.

\begin{tabular}{|l|l|l|l|l|}
\hline & \multicolumn{2}{|c|}{ IAE } & \multicolumn{2}{c|}{ TV } \\
\cline { 2 - 5 } & $Z_{I A E}[\mathrm{~m}]$ & $\theta_{I A E}[\mathrm{rad}]$ & $v_{T V}[\mathrm{~m} / \mathrm{s}]$ & $\omega_{T V}[\mathrm{rad} / \mathrm{s}]$ \\
\hline A. Original & 1,2154 & 0,6118 & 5,4019 & 7,0361 \\
\hline A. Nova & 1,1702 & 0,5081 & 5,4616 & 6,2998 \\
\hline
\end{tabular}

Tabela 1. Análise Quantitativa

retamente do plano da imagem, através de controladores NMPC, utilizando técnicas de visão computacional. Comparado à abordagem original, o novo algoritmo é capaz de estimar uma função quadrática para melhor representar o caminho, calcular a curvatura visual desejada a partir da função do caminho, além de lidar com ausências de trechos durante o trajeto e pisos de coloração não-homegênea. Através dos resultados experimentais é possível notar a superioridade do algoritmo proposto, onde foi possível seguir todo o percurso, mesmo na presença das imperfeições supracitadas, implicando no incremento da robustez. Para trabalhos futuros, pretende-se modificar o modelo de seguimento de caminhos visuais para inclusão de horizontes visuais variantes no tempo, uma vez que a técnica aqui apresentada gera funções exatas correspondentes ao caminho visual, e melhorar o algoritmos para lidar com cenários mais caóticos, encontrados em ambientes outdoor.

\section{REFERÊNCIAS}

Corke, P. (2011). Robotics, Vision and Control: Fundamental Algorithms in MATLAB, Springer Tracts in Advanced Robotics, Springer Berlin Heidelberg.

Kim, Z. (2008). Robust lane detection and tracking in challenging scenarios. IEEE Transactions on Intelligent Transportation Systems 9(1), pp. 16-26.

Kuo, Y.; Pai, N.; Li, Y. (2011). Vision-based vehicle detection for a driver assistance system . Computers and Mathematics with Applications, pp. 2096-2100

Lu, W.; Florez, S. A. R.; Seignez, E.; Reynaund, R. (2014). An Improved Approach for Vision-Based Lane Marking Detection and Tracking. International Conference on Electrical, Control and Automation Engineering, Hong Kong, China. DEStech Publications, Inc., pp.382-386.

Mammeri, A.; Boukerche, A.; Lu, G. (2014). Lane detection and tracking system based on the MSER algorithm, hough transform and kalman filter. MSWiM '14 Proceedings of the 17th ACM international conference on Modeling, analysis and simulation of wireless and mobile systems, pp. $259-266$
Wang, Y. ; Teoh, E.; Shen, D. (2004). Lane detection and tracking using B-Snake. Image and Vision Computing 22, pp. 269-280.

Chen, H.; Jin, Z. . (2010). Research on Real-Time Lane Line Detection Technology Based on Machine Vision, International Symposium on Intelligence Information Processing and Trusted Computing, Huanggang, pp. 528-531.

Sun, T.; Tsai, S.; Chan, V. . (2006). HSI color model based lane-marking detection, 2006 IEEE Intelligent Transportation Systems Conference, Toronto, Ont., pp. 1168-1172.

Arakeri, M. P., Kumar, B. P. V., Barsaiya, S. and Sairam, H. V. (2017). Computer vision based robotic weed control system for precision agriculture, 2017 International Conference on Advances in Computing, Communications and Informatics (ICACCI), pp. 1201-1205.

Gorbunov, V., Bobkov, V., Htet, N. W. and Ionov, E. (2018). Automated control system of fabrics parameters that uses computer vision, 2018 IEEE Conference of Russian Young Researchers in Electrical and Electronic Engineering (EIConRus), pp. 1728-1730.

Tianqi, L. (2017). A review of lane perception and automobile control based on computer vision, 2017 Second International Conference on Mechanical, Control and Computer Engineering (ICMCCE), pp. 6-11.

Ribeiro, T. T.; Fernandes, R. O. . C. A. G. S. (2016). Seguimento de caminhos visuais baseado em nmpc para robôs diferenciais, XXI Congresso Brasileiro de Automática - CBA2016.

Ribeiro, T. T.; Conceição, A. G. S. (2018a). Nonlinear Model Predictive Visual Path Following Control to Autonomous Mobile Robots. In: Journal of Intelligent Robotic Systems, 2018, pp. 1-13.

Ribeiro, T. T.; Fernandes, R. O. ; Conceição, A. G. S.; Franco, I. J. P. B. (2018b). Seguimento de Caminhos Visuais: Uma Abordagem Para Identificação de Parâmetros Através de Visão Computacional. In: XXII Congresso Brasileiro de Automática - CBA2018, 2018, João PessoaPB. XXII Congresso Brasileiro de Automática - CBA2018. 

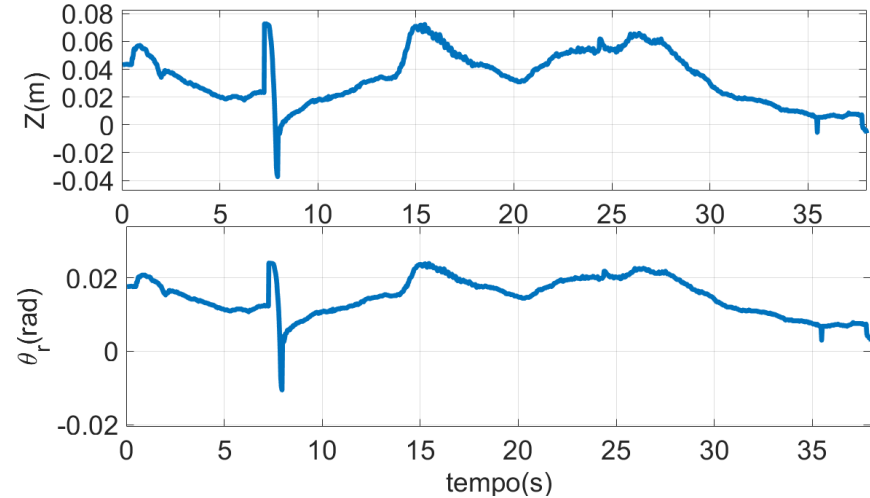

(a) Erros de Seguimento - Abordagem Original.
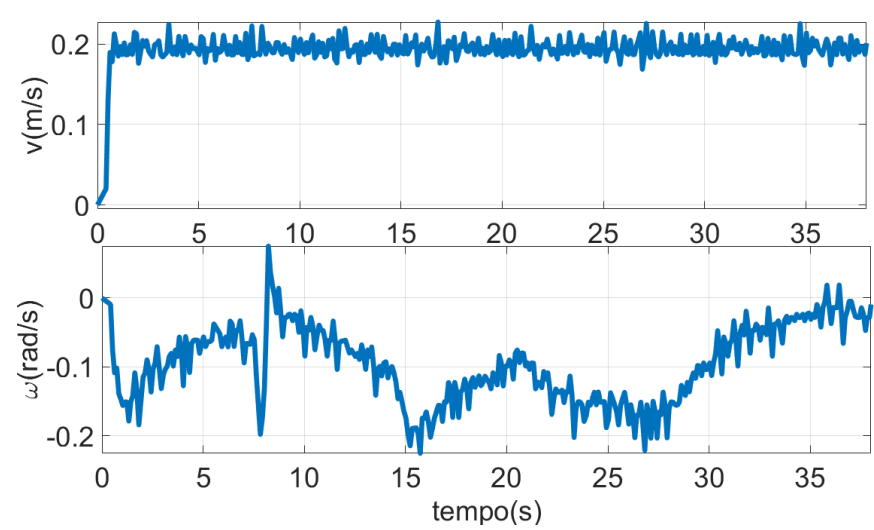

(c) Esforço de Controle - Abordagem Original.

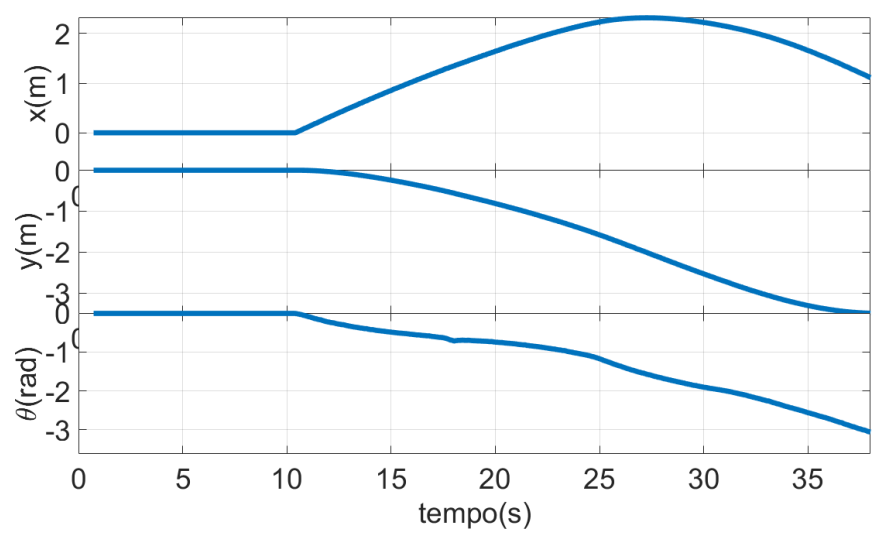

(e) Posturas - Abordagem Original.

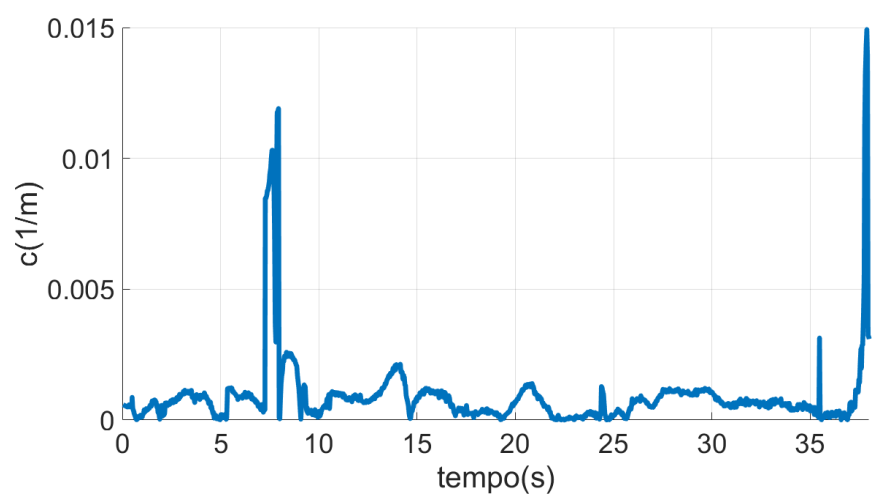

(g) Curvatura - Abordagem Original.

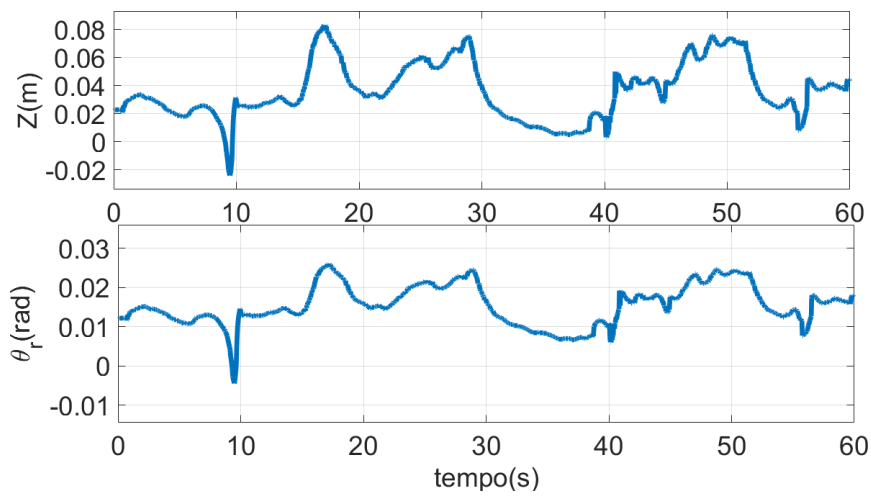

(b) Erros de Seguimento - Abordagem Nova.

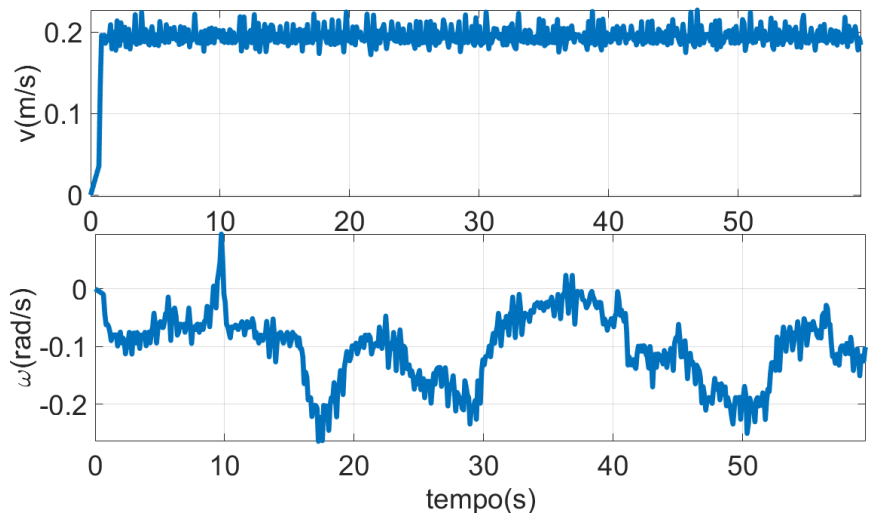

(d) Esforço de Controle - Abordagem Nova.

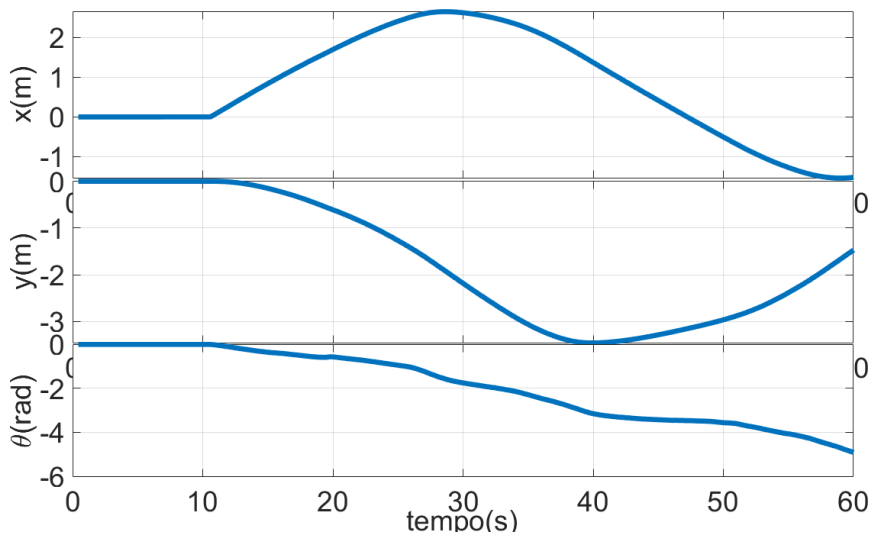

(f) Posturas - Abordagem Nova.

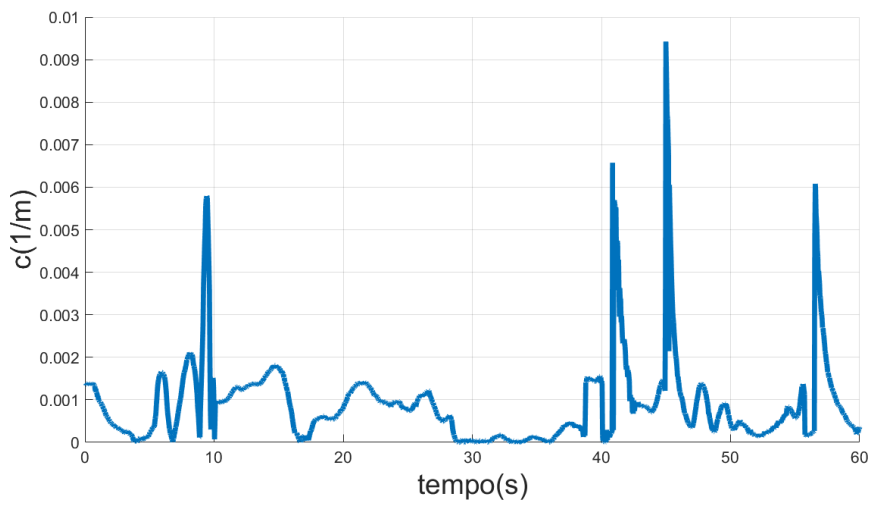

(h) Curvatura - Abordagem Nova.

Figura 9. Comparação das abordagens. 